\title{
Actualización en el diagnóstico y terapéutica en hipertensión pulmonar arterial
}

\author{
Mónica Zagolin $\mathrm{B}^{1}$, Eduardo Wainstein $\mathrm{G}^{3}$, Polentzi \\ U riarte $\mathbf{G}^{2}$.

\section{Update in the diagnosis and therapy for pulmonary arterial hypertension}

Pulmonary Arterial Hypertension includes a heterogeneous group of disorders with a common genetic, pathological and hemodinamyc origin. It is characterized by a high pulmonary artery pressure due to a primary vascular disease, as a consequence of genetic and environmental factors. The common pathway is a vascular imbalance towards vasoconstriction and proliferation inside the small vessels. According to the World Health Organization, 2003, Pulmonary Arterial Hypertension is classified as idiopathic, familiar or associated to connective tissue diseases, HIV, drugs, porto-pulmonary hypertension, congenital intracardiac shunts and others. The diagnosis is based in hemodynamics. Echocardiogram is a non invasive and right ventricular catheterization is an invasive diagnostic tool. Follow up is based on a clinical and functional assessment through functional class classification, dyspnea scores and 6-minute walking test. The prognosis is historically devastating but new therapies are changing the natural history of the disease. New treatments have demonstrated improvement in symptoms, hemodynamic profiles and survival. Intravenous, subcutaneous or inhaled prostanoids such as Epoprostenol, Treprostinil or lloprost respectively have been approved for Pulmonary Arterial Hypertension treatment as well as oral endothelial receptor blockers. They are all considered first line treatments for arterial pulmonary hypertensive patients with even better benefits than lung transplantation. Phosphodiesterase inhibitors (Sildenafil), have been recently approved for the treatment of pulmonary arterial hypertension (Rev Méd Chile 2006; 134: 902-9).

(Key words: Hypertension, pulmonary; Prostaglandins; Receptors, vascular endothelial growth factor; Sildenafil)

\footnotetext{
Recibido el 6 de mayo, 2005. Aceptado el 23 de diciembre, 2005.

${ }^{1}$ Departamento de Medicina del Instituto Nacional del Tórax. ${ }^{2}$ Departamento de Cardiología del Instituto Nacional de Tórax. ${ }^{3}$ Departamento de Medicina, Clínica Las Condes y Escuela de Medicina, Universidad Andrés Bello.
}

La a hipertensión pulmonar arterial (HPA) es una entidad poco frecuente, de evolución progresiva y devastadora, que afecta principalmente a

Correspondencia a: Mónica Zagolin Blancaire. José Manuel Infante 717, Providencia, Santiago. E mail: mzagolin@torax.cl mujeres jóvenes en plena edad productiva. Se ha comunicado, para HPA primaria o idiopática, según nueva nomenclatura, una incidencia de 1-2 pacientes nuevos por millón de habitantes por año ${ }^{1}$. Su etiología es desconocida, pero parece ser el resultado de una interacción anormal entre 
factores genéticos y medioambientales, que llevan finalmente a un daño vascular centrado en un desequilibrio endotelial a favor de la vasoconstricción y proliferación vascular. Desde un punto de vista histopatológico, el compromiso vascular va desde hipertrofia de la íntima y capa media de la arteriola pulmonar, asociada a trombosis in situ, hasta lesiones plexiformes fibróticas en su etapa terminal 2,3 .

Aproximadamente, 6\% sigue una distribución familiar, con una relación similar hombre-mujer y de transmisión autosómica dominante con penetrancia incompleta, es decir, suele saltar generaciones. La presencia del gen no implica desarrollo de la enfermedad $^{4}$ y es así como uno de los genes identificados como responsables de la enfermedad, conocido como PPH1 (locus 2q 31-32), tiene una expresión clínica en menos de $20 \%$ de los portadores $^{5}$. Recientemente, se ha correlacionado a las mutaciones en el gen II del receptor de la proteína morfogenética ósea (BMPR II) con las lesiones vasculares proliferativas observadas en los pacientes con HPA, sugiriendo que se trataría del mismo gen $\mathrm{PPH}^{6}$.

La HPA afecta con mayor frecuencia a las mujeres en una relación 1,7:1 y, comúnmente, se expresa en la cuarta década de la vida. El diagnósti- co habitualmente es efectuado entre 18 y 24 meses posterior al inicio de los síntomas, debido al carácter inespecífico de ellos, tales como disnea, fatiga 0 dolor torácico, de modo que es frecuente pesquisar enfermos con severa limitación funcional y estrecho margen terapéutico. La sobrevida histórica promedio es de 2,8 años luego del diagnóstico ${ }^{1,7,8}$.

Se define HPA mediante el estudio hemodinámico (cateterismo derecho) ante la presencia de presión media de la arteria pulmonar (PAPM) $>25$ $\mathrm{mmHg}$ en reposo o $>30 \mathrm{mmHg}$ en ejercicio, acompañado de resistencia vascular pulmonar (RVP) $>160$ dinas/seg $\mathrm{x} \mathrm{cm}^{-5}$ 1,5.

\section{CiASIFICACIÓN}

La Organización Mundial de la Salud (OMS), en 1998, reclasificó la hipertensión pulmonar $(\mathrm{HP})^{5}$, efectuando algunas modificaciones en $2003^{9}$ que se ilustran en la Tabla 1. Estas apuntan a sugerir el uso del término HPA idiopática (sin causa subyacente) en vez de primaria, término usado desde 1951 por Dresdale ${ }^{10}$, y además se incorporó en este grupo a la enfermedad veno-oclusiva y la angiomatosis capilar pulmonar.

Tabla 1. Clasificación actual de la Hipertensión Pulmonar de la O rganización M undial de la Salud (2003)

I. Hipertension Arterial Pulmonar

- Idiopática

- Familiar

- Asociada a: colagenopatías, cortocircuitos intracardiacos, hipertensión portal; virus inmunodeficiencia humana, drogas y toxinas, hipertiroidismo, enfermedad de Gaucher, hemoglobinopatías, desórdenes mieloproliferativos, telangectasia hereditaria familiar, esplenectomía.

- Enfermedad veno-oclusiva.

- Angiomatosis capilar pulmonar

II. Hipertensión Venosa Pulmonar

- Asociada a aumento de presión ventricular y/o auricular izquierda, enfermedad valvular izquierda.

III. Asociada a Enfermedad Pulmonar o Hipoxemia

- Enfermedad pulmonar obstructiva crónica, enfermedad pulmonar intersticial, trastornos del sueño, síndrome de hipoventilación alveolar.

IV. Asociada a Enfermedad Tromboembólica Crónica

- Tromboembolia arterial pulmonar proximal.

- Obstrucciones distales en arterias pulmonares.

V. Misceláneas

- $\quad$ Sarcoidosis, histiocitosis X, linfangioleiomiomatosis 
En la categoría «HP asociada a» se encuentran las enfermedades colágeno vasculares tales como esclerodermia, lupus, enfermedad mixta del tejido conectivo, dermatopolimiositis, artritis reumatoide y síndrome de Sjögren 1,3,5,9. La entidad más frecuentemente asociada es la esclerosis sistémica limitada, variante CREST. La prevalencia de esta asociación es variable, sin embargo, recientemente se ha estimado, mediante estudio hemodinámico invasivo, cercana a $12 \% 11$. En este grupo también se encuentran los pacientes con HIV $(0,5 \%)^{12}$, usuarios de drogas anorexígenas (aminorex, fenfluramina, dexfenfluramina) ${ }^{13}$, con hipertensión portal $(2-5 \%)^{14}$ y comunicaciones intracardiacas congénitas de derecha a izquierda (prevalencias alrededor de 50\% para los defectos ventriculares y $10 \%$ para defectos auriculares) ${ }^{15}$.

\section{Diagnóstico}

El diagnóstico clínico es tardío. Suele manifestarse con disnea progresiva, asociada a fatigabilidad, dolor torácico, mareo, palpitaciones y, ocasionalmente, a lipotimia o síncopes, siendo este último síntoma un marcador de mal pronóstico. Los estadios más avanzados son identificados por signos y síntomas secundarios a la falla ventricular derecha ${ }^{3,5,9}$.

En la evaluación diagnóstica se sugiere efectuar un estudio serológico que incluya detección de VIH, factor reumatoideo, anticuerpos anticardiolipinas, anticuerpos antinucleares (ANA) y anticuerpos antifracciones extractables del núcleo (ENA: Ro, La, Sm, RNP y Jo-1). Además, se recomienda efectuar un perfil hepático, de coagulación, tiroideo y test de embarazo. El estudio funcional respiratorio que incluye una espirometría, estudio de difusión y volúmenes, permite investigar o descartar entidades asociadas al compromiso parenquimatoso pulmonar? ${ }^{3}$.

El ecocardiograma es el mejor examen en el inicio del estudio de pacientes con sospecha de HP, permitiendo la medición de la presión arterial pulmonar sistólica (PAPS), función del ventrículo derecho y la detección de cortocircuitos intracardíacos. El nivel de precisión de la ecocardiografía es variable, por lo que es fundamental en todo paciente efectuar un estudio hemodinámico confirmatorio. En operadores expertos, la PAPS que puede ser estimada en 59 a $72 \%$ de los pacientes ${ }^{16,17}$.
El test de caminata de 6 min es un examen simple y no invasivo, de extraordinaria utilidad en la evaluación inicial de los pacientes con HPA, en el seguimiento y en el análisis de la respuesta terapéutica. Se ha reportado una excelente comelación pronóstica entre la distancia recomida y la evolución a largo plazo, documentándose una peor evolución en aquellos que caminan menos de $332 \mathrm{mt}^{16,18}$.

La radiografía de tórax puede ser normal hasta en $10 \%$. Clásicamente, se observa prominencia de las arterias pulmonares (90\%) y amputación periférica de los vasos. El cintigrama de ventilación perfusión, generalmente, muestra un patrón de perfusión irregular difuso, caracterizado por múltiples defectos subsegmentarios. Su principal aporte se encuentra en el estudio de pacientes con enfermedad tromboembólica crónica (ETC), en la que se observa grandes defectos de perfusión. En esta patología, la tomografía axial computada con contraste permite una adecuada visualización de las arterias pulmonares centrales, presencia de trombos e información adicional sobre el estado del parénquima pulmonar. En los casos en que se sospecha ETC, la resonancia nuclear magnética y la angiografía suelen ser de ayuda complementaria $3,5,9$.

Finalmente, el diagnóstico de certeza lo otorga el estudio hemodinámico a través de la cateterización de arteria pulmonar con catéter de Swan-Ganz y medición precisa de la presión de arteria pulmonar, capilar pulmonar, gasto cardíaco y oximetrías sectoriales. Este permite, además, evaluar la vaso-reactividad pulmonar, que puede efectuarse mediante la administración de adenosina (usada en nuestro medio en dosis hasta $250 \mathrm{mcg} / \mathrm{kg} / \mathrm{min}$ ), óxido nítrico (ON) inhalado (18 partes por millón) o epoprostenol (20 ng/ $\mathrm{kg} / \mathrm{min}$ i.v. $)^{1,5}$. Clásicamente, se ha considerado una respuesta positiva a una disminución del orden de $20 \%$ en la PAPM o la resistencia vascular pulmonar (RVP) sin caída del débito cardíaco ${ }^{3}$, aunque actualmente se considera un resultado positivo a una disminución de la PAPM o RVP a cifras más cercanas a lo normal (PAPM inferior a $40 \mathrm{mmHg}$ ). Esta condición sugiere un mejor pronóstico y una respuesta favorable a la terapia con bloqueadores de canales de calcio $(\mathrm{BCC})^{19}$. En la expeniencia de expertos, no más allá de $10-12 \%$ de los pacientes con HPA idiopática son vaso-reactivos. En atención a estos resultados es que hoy se desaconseja el uso empírico de $\mathrm{BCC}^{20}$.

El seguimiento y control de la terapia es clínico y funcional. La escala más usada en la 
evaluación de la capacidad funcional es la de la OMS, muy similar a la New York Heart Association (NYHA), que considera no sólo disnea, sino también la presencia de síncopes, dolor torácico y fatiga y separa en estadios I a IV ${ }^{5}$. La disnea es evaluada fundamentalmente a través de la puntuación de Borg (de 0 a 10), siendo 10 el grado máximo de disnea. La distancia recorrida medida en el test de caminata de 6 min es el examen, por excelencia, para el seguimiento de estos pacientes por su sencillez, reproducibilidad y adecuada correlación pronóstica ${ }^{16,18}$. El estudio hemodinámico, como método de control de la terapia establecida, es usado principalmente cuando existe un protocolo de investigación en curso o un deterioro clínico funcional pese al tratamiento.

\section{HPA Y EMBARAZO}

Constituye un aspecto relevante de tratar, ya que la mortalidad de un embarazo en una paciente con HPA fluctúa entre 30 y $56 \%{ }^{21}$; esto, sin considerar el riesgo de teratogenicidad asociada a terapias tales como antiendotelinas o anticoagulantes. Se debe aconsejar un método anticonceptivo definitivo y seguro. No es recomendable el uso de anticonceptivos orales o dispositivos intrauterinos por los riesgos inherentes (trombogénesis, hemorragia).

\section{TRATAMIENTO}

La HPA afecta la calidad de vida de los pacientes en forma progresiva y sólo se ha descrito remisión espontánea en casos aislados, asociados al uso y posterior suspensión de anorexígenos ${ }^{13}$.

Por muchos años, las únicas alternativas terapéuticas disponibles se basaron en anticoagulación, $\mathrm{BCC}$, diuréticos y oxígeno en casos específicos y desde la década 1990-99, el trasplante pulmonar 0 cardiopulmonar. La anticoagulación ha sido recomendada en todos los pacientes con HPA, en base a dos pequeños estudios que sugieren que su uso mejora la sobrevida, sustentados en los hallazgos histopatológicos de trombosis in situ y trombogénesis aumentada, ambos elementos que promueven la progresión de la enfermedad 3,9 .

En HPA se considera que un tratamiento es efectivo si es capaz de prolongar sobrevida, aliviar síntomas, mejorar capacidad funcional y al mismo tiempo si es seguro, fácil de usary de costo razonable.

En los últimos 10 años se han desarrollado fármacos que han cambiado la historia natural de la enfermedad, lo que ha permitido anteponer estas terapias al trasplante y se ha generado una verdadera explosión de nuevas alternativas terapéuticas que a continuación se detallan y se ilustran en la Tabla 2.

\section{BLOQUeADORES DE LOS CANALES DE CALCIO}

Son sólo una alternativa útil y económica para un restringido grupo de enfermos en los que el test de vaso-reactividad es positivo ${ }^{19}$, con un beneficio a largo plazo limitado ${ }^{20}$. Los BCC tales como diltiazem o amlodipino están indicados en pacientes en clase funcional WHO II o III y su uso suele estar limitado por los efectos adversos asociados a las elevadas dosis requeridas del orden de $960 \mathrm{mg}$ de diltiazem o $240 \mathrm{mg}$ de nifedipino, tales como eritema facial, edema o hipotensión ${ }^{1}$.

\section{PROSTACICLINAS}

El uso de prostaciclinas endovenosas (iv) tales como epoprostenol o iloprost ha demostrado beneficios tanto sintomáticos, hemodinámicos y en la sobrevida a corto y largo plazo en los pacientes con HPA. La terapia con prostaciclinas vía iv es considerada la mejor altemativa terapéutica disponible para los pacientes en clase funcional III y IV; sin embargo, no está exenta de toxicidad, es de alto costo y requiere un alto nivel de comprensión y colaboración de parte del paciente. Ella involucra el uso de un catéter permanente, ya sea endovenoso o subcutáneo con su respectiva bomba infusora portátil o el uso de nebulizadores ultrasónicos, para uso por la vía inhalatoria ${ }^{3}$.

Epoprostenol. Es una prostaglandina sintética (PGI2), cuyo efecto vasodilatador selectivo pulmonar fue evidenciado por Higgenbottam en la década 1980-89. Desde entonces, numerosos estudios han demostrado significativos beneficios en el alivio sintomático, mejonía funcional, hemodinámica y en la sobrevida ${ }^{22}$. El beneficio de epoprostenol (Flolan ${ }^{\circledR}$ ) no sólo se debe a sus propiedades vasodilatadoras, ya que además disminuye la agregación y migración plaquetaria y posee propiedades antiproliferativas. Epopros- 
Tabla 2. Comparación entre los diferentes ensayos terapéuticos y drogas anti-H PA

\begin{tabular}{|c|c|c|c|c|c|}
\hline Terapia & Autor/Año & Tipo de estudio & «»» & Seguimiento & Resultados \\
\hline $\begin{array}{l}\text { Epoprostenol } \\
\text { endovenoso }\end{array}$ & $\begin{array}{l}\text { Barst } \\
1996\end{array}$ & RP-DC-CP & 81 & 12 semanas & $\begin{array}{l}\text { Mejoría sintomática, } \\
\text { hemodinámica, } \\
\text { distancia (47 mt) } \\
\text { y sobrevida }\end{array}$ \\
\hline $\begin{array}{l}\text { Treprostinil } \\
\text { subcutáneo }\end{array}$ & $\begin{array}{l}\text { Simonneau } \\
2002\end{array}$ & M-RP-DC-CP & 470 & 12 semanas & $\begin{array}{l}\text { Mejoría sintomática, } \\
\text { distancia (16 mt) } \\
\text { y hemodinámica }\end{array}$ \\
\hline $\begin{array}{l}\text { Iloprost } \\
\text { inhalado }\end{array}$ & $\begin{array}{l}\text { Olshewski } \\
2002\end{array}$ & M-RP-DC-CP & 203 & 12 semanas & $\begin{array}{l}\text { Mejoría sintomática, } \\
\text { distancia ( } 36 \mathrm{mt}) \\
\text { y hemodinámica }\end{array}$ \\
\hline $\begin{array}{l}\text { Bosentan } \\
\text { Oral }\end{array}$ & $\begin{array}{l}\text { Rubin } \\
2002\end{array}$ & M-RP-DC-CP & 213 & 16 semanas & $\begin{array}{l}\text { Mejoría sintomática } \\
\text { distancia ( } 44 \mathrm{mt}) \\
\text { y hemodinámica }\end{array}$ \\
\hline $\begin{array}{l}\text { Sildenafil } \\
\text { Oral }\end{array}$ & $\begin{array}{l}\text { Sastri } \\
2004\end{array}$ & $\begin{array}{l}\mathrm{RP}-\mathrm{CP} \\
\text { Cross-over }\end{array}$ & 22 & 6 semanas & $\begin{array}{l}\text { Mejoría sintomática, } \\
\text { mejoría test } \\
\text { ejercicio } \\
\text { (Naughton), } \\
\text { calidad de vida }\end{array}$ \\
\hline
\end{tabular}

n: Tamaño de la muestra. M: Multicéntrico. RP: Randomizado, prospectivo. DC: Doble ciego. CP: Control placebo.

tenol es el tratamiento de elección para los pacientes en clase funcional III y IV, por su beneficio ampliamente demostrado en la sobrevida a largo plazo en seguimientos que superan los 10 años ${ }^{23}$. La sobrevida reportada con epoprostenol a 1, 3 y 5 años es de 85\%, $63 \%$ y $55 \%$, respectivamente, comparada con $58 \%$, $33 \%$ y $28 \%$, en el mismo tiempo, en los controles 24 . Su uso está aprobado por las sociedades europea y americana de la especialidad.

Sus beneficios han sido reportados en HPA idiopática, familiar, asociada a drogas o esclerodermia, shunts debido a malformaciones congénitas, hipertensión portal, sarcoidosis, HIV y en tromboembolismo pulmonar crónico no quirúrgico ${ }^{25}$. El elevado costo ha obstaculizado su disposición a nivel nacional y el interés se ha volcado hacia nuevas alternativas más económicas. Entre ellas, se encuentran las prostaglandinas inhaladas (iloprost), orales (beraprost), subcutáneas (treprostinil), los bloqueadores de receptores de endotelinas tipo I (bosentan) y los antifosfodiesterasa V (sildenafil).

Iloprost inhalado. Es una prostaciclina análoga para uso inhalatorio. Su beneficio a corto y largo plazo ha sido demostrado en un estudio multicéntrico, prospectivo, randomizado contra placebo, tanto del punto de vista sintomático y funcional como hemodinámico, por lo que se recomienda para pacientes en clase funcional III y IV ${ }^{26}$. Su vida media reducida exige un uso repetido entre 6 y 9 veces al día, entre 5 y 15 min cada vez ${ }^{26}$. Su uso está aprobado por las sociedades europea y americana de la especialidad.

Beraprost. Análogo oral, tiene una vida media más larga que epoprostenol; sin embargo, sus resultados provienen de pequeños estudios en HPA idiopática y asociada a enfermedad cardíaca congénita, con mejoría hemodinámica sólo a corto plazo $^{27}$. Por el momento, se requiere mayor investigación al respecto para su recomendación. Sólo se encuentra aprobada en Japón.

Treprostinil. Prostaciclina de uso subcutáneo (Remodulin ${ }^{\circledR}$ ), en infusión continua. Treprostinil ha demostrado tener un beneficio no sólo sintomático, sino también en las variables funcionales y hemodinámicas en un gran estudio multicéntrico, randomizado, prospectivo ${ }^{28}$. Si bien su uso es más 
simple que su símil endovenoso, su costo sigue siendo elevado y presenta una elevada tasa de abandono prematuro por dolor en la pared abdominal en el sitio de infusión (8\%). Sólo aprobado para su uso en Estados Unidos de Norteamérica (EE.UU) y en algunos países de Europa.

Bosentan. Bosentan $\left(\right.$ Tracleer $\left.^{\circledR}\right)$ es la primera droga oral aprobada en EE.UU, Canadá y Suiza para el tratamiento de la HPA. El bloqueo dual de los receptores A y B de endotelina (ET-1) es una nueva y revolucionaria alternativa terapéutica, que ha demostrado efectividad clínica, funcional y hemodinámica en los pacientes con HPA idiopática y asociada a ETC ${ }^{29}$. El único efecto adverso de significación es su potencial hepatotoxicidad (2\%), con reversibilidad absoluta luego del retiro de la droga. Su uso está aprobado en EE.UU y Europa para clase funcional II, III y IV y se ha constituido, junto con epoprostenol, en la terapia de elección en el paciente con HPA. Se encuentra contraindicado en el embarazo o en asociación a ciclosporina A o glyburide.

$\mathrm{Si}$ bien el bloqueo dual de receptores de endotelina es beneficioso, los ET1-B se expresan predominantemente en la célula endotelial, donde su activación estimula la liberación de agentes como ON y prostaciclina bajo condiciones fisiológicas normales, por lo que parece razonable el intento de un bloqueo más selectivo de receptores de ET1. Actualmente se encuentran en proceso de evaluación dos bloqueadores específicos ET1-A tales como ambrisentan y sitaxsentan.

Sildenafil. Es un bloqueador altamente específico de la enzima fosfodiesterasa 5, ampliamente distribuida en el territorio pulmonar, cuya función es inhibir a cGMP, el segundo mensajero del ON. Sildenafil, al bloquear esta enzima, previene la caída de cGMP y favorece una mayor disponibilidad de $\mathrm{ON}$ en la vasculatura pulmonar y su consecuente efecto vasodilatador pulmonar selectivo.

Existen muchas comunicaciones y series clínicas pequeñas que comunican resultados beneficiosos. Hasta el momento, el único ensayo randomizado publicado que demuestra su beneficio, fue efectuado en 22 pacientes con modalidad cross-over, con 6 semanas de seguimiento y control con técnicas no invasivas ${ }^{30}$. Los resultados del estudio multicéntrico Super-1 han sido comu- nicados en los últimos congresos de la especialidad y son favorables.

En un estudio en agudo, comparando sildenafil (Viagra ${ }^{\circledR}$ ) con ON, se demostró una caída significativa de la presión de arteria pulmonar con sildenafil ${ }^{31}$. También se ha reportado su beneficio en un grupo selecto de pacientes con ETC no quirúrgica, con resultados favorables en relación a la capacidad de ejercicio y hemodinamia ${ }^{32}$.

Otros. Los resultados con drogas como ß-bloqueadores, agonistas $\alpha$-adrenérgicos, nitratos, hidralazina 0 inhibidores de la enzima de conversión de angiotensina han sido desalentadores. Se encuentran aún en evaluación alternativa como L-Arginina (sustrato para la síntesis de $\mathrm{ON}$ ), estatinas, antiendotelinas específicos entre otras 3,9 .

Septostomía. En pacientes extremadamente graves, refractarios a terapia médica y como un puente al trasplante, se ha visto un efecto beneficioso a corto plazo con la creación de un defecto auricular septal artificial (septostomía), que permite aliviar la carga al ventrículo derecho, mejorando el débito cardiaco con el costo de una inmediata caída de la saturación arterial ${ }^{33}$.

Trasplante pulmonar (TP). HPA es una de las indicaciones clásicas de TP; sin embargo, con la aparición de nuevas terapias, en la actualidad da cuenta de sólo 3,8\% del total de los TP. Esto se explica por las cifras de sobrevida alcanzadas a 1, 3 y 5 años de $65 \%$, 55\% y 44\%, inferiores a las reportadas con epoprostenol ${ }^{34}$. En los pacientes con cardiopatías congénitas complejas es preferido el bloque corazón-pulmón; sin embargo, en los otros grupos no hay acuerdo respecto al trasplante de pulmón único o bilateral, el primero asociado a mayor mortalidad precoz, el segundo conllevaría una menor disponibilidad de órganos ${ }^{35}$.

\section{PRONÓSTICO}

Son indicadores de mal pronóstico: clase funcional de la OMS IV, distancia recorrida inferior a 332 $\mathrm{m}$, presión arterial pulmonar media superior a 55 $\mathrm{mmHg}$, presión de aurícula derecha superior a 10 $\mathrm{mmHg}$, índice cardíaco inferior a $2,0 \mathrm{l} / \mathrm{min} / \mathrm{m}^{2} \mathrm{y}$ saturación arterial pulmonar inferior a $63 \%{ }^{8}$. 


\section{SOBREVIDA}

El registro norteamericano de sobrevida demuestra que la historia natural de la enfermedad es progresiva y devastadora. Es así como los pacientes en clase funcional I y II tienen una sobrevida promedio de 58 meses, comparada con menos de 6 meses en los en clase IV ${ }^{7}$.

La causa de muerte es una progresiva falla ventricular derecha y brusca caída del débito cardíaco ${ }^{3,5,7,9}$.

\section{EXPERIENCIA NACIONAL}

En el sistema público de salud, sólo se dispone de bloqueadores de los canales de calcio. En algunos centros de referencia, de alta complejidad, se dispone de prostaciclinas endovenosas o inhalato-

\section{REFERENCIAS}

1. The International Primary Pulmonary Hypertension Study Group. The International Primary Pulmonary Hypertension Study (IPPHS). Chest 1994; 105 (Suppl): 37S-41S.

2. RuBin LJ. Primary pulmonary hypertension. Chest 1993; 104: 236-50.

3. British Cardiac Society Guidelines and Medical Practice Committee, and approved by the British Thoracic Society and the British Society of Rheumatology. Recommendations on the Management of Pulmonary Hypertension in Clinical Practice Heart 2001; Volume 86 (Supplement 1): i1-i13.

4. LANGLeBEN D. Familial primary pulmonary hypertension. Chest 1994; 105: 13-6S.

5. RiCH S, EDITOR. Primary pulmonary hypertension. Executive Summary from the World Symposium. Primary Pulmonary Hypertension. World Health Organization, 1998.

6. Deng Z, Morse JH, Slager SL, Cuervo N, Moore KJ, Venetos G et AL. Familial primary pulmonary hypertension (gene PPH 1) is caused by mutations in the bone morphogenetic protein receptor-II gene. Am J Hum Genetics 2000; 67: 737-44.

7. Rich S, Dantzker DR, Ayres SM, Bergofsky EH, Brundage BH, Detre KM et al. Primary pulmonary hypertension: a national prospective study. Ann Intern Med 1987; 107: 216-23. rias, inhibidores de fosfodiesterasa y posibilidad de reclutamiento en protocolos fase III con ambrisentan. La experiencia publicada hasta la fecha se limita exclusivamente al reporte de casos especiales, como el de HP y embarazo ${ }^{36}$.

La experiencia del Instituto Nacional del Tórax en el estudio y tratamiento de estos pacientes será comunicada próximamente. A modo preliminar, es posible señalar que en el lapso de dos años, el diagnóstico de HPA se ha confirmado en 41 pacientes, la mayoría mujeres, jóvenes, en capacidad funcional III con HPA moderada. En 20 pacientes tratados con sildenafil por 6 meses se observó una mejonía clínica y funcional significativa.

Finalmente recomendamos que los pacientes sean referidos lo antes posible, sin inicios empíricos de terapias específicas, a centros de mayor complejidad con experiencia en esta entidad.

8. D’Alonzo GE, Barst RJ, Ayres SM, Bergofsky EH, Brundage BH, Detre KM et al. Survival in patients with primary pulmonary hypertension: results from a national prospective registry. Ann Intern Med 1991; 115: 343-9.

9. Simonneau G, Galié N, Rubin LJ, Langleben D, Seeger W, Domenighetti G ET aL. Clinical classification of pulmonary hypertension. J Am Coll Cardiol 2004; 43: 5S-12S.

10. Dresdale D, Schultz M, Michton R. Primary pulmonary hypertension: I. Clinical and Hemodynamic study. Am J Med 1951; 11: 686-94.

11. Mukerjee D, St. George D, Coleiro B, Knight C, Denton CP, Davar J et al. Prevalence and outcome in systemic sclerosis associated pulmonary arterial hypertension: application of a registry approach. Ann Rheum Dis 2003; 62: 1088-93.

12. Speich R, Jenni R, Opravil M. Primary pulmonary hypertension in HIV infection. Chest 1991; 100: 1268-71.

13. Abenhaim L, Moride Y, Brenot F, Rich S, Benichou J, Kurz X et al, for the International Primary PulmoNARY HyPERTENSION Study Group. Appetite suppressant use and the risk of primary pulmonary hypertension. N Engl J Med 1996; 335: 609-16.

14. Hadengue A, Benhayoun MK, Lebrec D, Benhamou JP. Pulmonary hypertension complicating portal hypertension: prevalence and relation to splachnic hemodynamic. Gastroenterology 1991; 100: 520-8 
15. Kidd L, Driscoll DJ, Gersony WM, Hayes CJ, KeANe JF, O’FALON WM ET AL. Second natural history study of congenital heart defects. Results of treatment of patients with ventricular septal defects. Circulation 1993; 87(2 Suppl): 138-51.

16. McGoon M, Gutterman D, Sten V, Barst R, McCRORY DC, Fortin TA ET AL. Screening, early detection, and diagnosis of pulmonary arterial hypertension. ACCP Evidence-Based Clinical Practice Guidelines. Chest 2004; 126: 14S-34S.

17. Berger M, Haimowitz A, Van Tosh A, Berdoff RL, GOLDBERG E. Quantitative assessment of pulmonary hypertension in patients with tricuspid regurgitation using continuous wave Doppler ultrasound. AJ Am Coll Cardiol 1985; 6: 359-65.

18. Miramoto S, Nagaya N, Satoh T, Kyotani S, SakamaKI F, FuJITA M ET AL. Clinical correlates and prognostic significance of six-walk test in patients with primary pulmonary hypertension. Am J Respir Crit Care Med 2000; 161(2 Pt 1): 487-92.

19. Rich S, BRuNdage BH. High-dose calcium channelblocking therapy for primary pulmonary hypertension: evidence of long-term reduction in pulmonary arterial pressure and regression of right ventricular hypertrophy. Circulation 1987; 76: 135-41.

20. RuBIN LJ, BADESCH DB. Evaluation and management of the patient with pulmonary arterial hypertension. Ann Intern Med 2005; 143: 282-92.

21. Weiss BM, Zemp L, SeIFerT B, Hess OM. Outcome of pulmonary vascular disease in pregnancy: a systematic overview from 1978 through 1996. J Am Coll Cardiol 1998; 31: 1650-7.

22. Barst RJ, Rubin LJ, Long WA, McGoon MD, Rich S, BADESCH DB ET AL. A comparison of continuous intravenous epoprostenol (Prostacyclin) with conventional therapy for primary pulmonary hypertension. N Engl J Med 1996; 334: 296-301.

23. Higgenbottam T, Siddons T. Trials of inhaled iloprost and other new vasodilating prostaglandins. Eur Respir J 2000; 17: 6-7.

24. Sitbon O, Humbert M, Nunes H, Parent F, García G, HeRve $P$ ET AL. Long-term intravenous epoprostenol infusion in primary pulmonary hypertension: prognostic factors and survival. J Am Coll Cardiol 2002; 40: 780-8.

25. McLaughun VV, Genthner DE, Panela MM, Rich S. Reduction in pulmonary vascular resistance with long-term epoprostenol (prostacyclin therapy in primary pulmonary hypertension). N Engl J Med 1998; 338: 273-7.

26. Olschewski H, Simonneau G, Nazzareno G, HigenbotTam T, NaEje R, Rubin LJ et al. Inhaled Iloprost for severe pulmonary hypertension. N Engl J Med 2002; 347: 322-9.

27. Barst RJ, McGoon M, McLaughlin V, Tapson V, Oudiz R, Shapiro S Et al. Beraprost Study Group. Beraprost therapy for pulmonary arterial hypertension. J Am Coll Cardiol 2003; 41: 2119-25.

28. Simonneau G, Barst RJ, Gale N, Naeje R, Rich S, Bourge RC ET AL. Continuous subcutaneous infusion of treprostinil, a prostacyclin analogue, in patients with pulmonary arterial hypertension: a double-blind, randomized, placebo-controlled trial. Am J Respir Crit Care Med 2002; 165: 800-4.

29. Rubin LJ, Badesch DB, Barst RJ, Galle N, Biack CM, KEOGH A ET AL. Bosentan therapy for pulmonary arterial hypertension. NEngl J Med 2002; 346: 896-903.

30. Sastry BKS, Narasimhan C, Krishna Reddy N, Soma Raju B. Clinical efficacy of sildenafil in primary pulmonary hypertension: a randomized, placebo-controlled, double-blind, crossover study. JACC 2004; 43: 1149-53.

31. Michelakis E, TymchaK W, Lien D, Webster L, НASнimoto K, ARcheR S. Oral sildenafil is an effective and specific pulmonary vasodilator in patients with pulmonary arterial hypertension. Circulation 2002; 105: 2398-403.

32. Ghofrani HA, Schermuly RT, Rose F, Wiedemann R, Kohstall MG, KReckel A et al. Sildenafil for longterm treatment of nonoperable chronic thromboembolic pulmonary hypertension. Am J Respir Crit Care Med 2003; 167: 1139-41.

33. Rich S, Dodin E, McLaughun VV. Usefulness of atrial septostomy as a treatment for primary pulmonary hypertension and guidelines for its application. Am J Cardiol 1997; 80: 369-71.

34. United Network for Organ Shaning. US scientific registry for transplant recipients and the organ procurement and transplantation network: transplant data: 1990-2000. Annual report. Washington, US Department of Health and Human Services, 2002.

35. Meyers BF, Lynch J, Trulock EP, Guthrie TJ, Cooper JD, Patterson GA. Lung transplantation: a decade of experience. Ann Surg 1999; 230: 362-70.

36. Valdés G, Matthei R, Fernández MS, Schacht C, Corthorn J, Germain A. Hipertensión pulmonar y embarazo. Rev Méd Chile 2002; 130: 201-8. 